\begin{tabular}{|c|l|}
\hline Title & Scaling behaviours in settling process of fractal aggregates in water \\
\hline Author(s) & Tian, W. J.; Nakay ama, T.; Huang, J. P.; Y u, K. W. \\
\hline Citation & $\begin{array}{l}\text { Europhysics Letters, 78(4), 46001 } \\
\text { https://doi.org/40.1209/0295-5075/78/46001 }\end{array}$ \\
\hline Issue Date & 2007 \\
\hline Doc URL & http://hdl.handle.net/2115/28253 \\
\hline Type & article \\
\hline File Information & EPL 78-4.pdf \\
\hline
\end{tabular}

Instructions for use 


\title{
Scaling behaviours in settling process of fractal aggregates in water
}

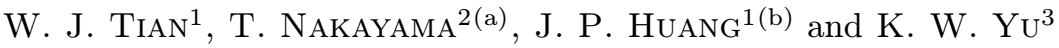 \\ ${ }^{1}$ Surface Physics Laboratory and Department of Physics, Fudan University - Shanghai 200433, China \\ ${ }^{2}$ Department of Applied Physics, Hokkaido University - Sapporo 060-8628, Japan \\ ${ }^{3}$ Department of Physics and Institute of Theoretical Physics, Chinese University of Hong Kong - Shatin, \\ New Territories, Hong Kong, China
}

received 22 February 2007; accepted in final form 4 April 2007

published online 3 May 2007

PACS 61.43.Hv - Fractals; macroscopic aggregates (including diffusion-limited aggregates)

PACS 47.57.-s - Complex fluids and colloidal systems

PACS 92.20.Vn - Sedimentation

\begin{abstract}
We investigate the effect of permeant flow on the sedimentation of porous fractalaggregates in water. Our theoretical analysis gives explicit calculations on the scaling behaviours of settling velocities, taking into account the fractality through a proper permeability for fractal aggregates. The calculated results for the scaling behaviours of settling velocities fit remarkably well with experimental data. The analytic expression for the settling velocity provides a criterion for determining fractal dimensions $D_{f}$ of aggregates from sedimentation experiments.
\end{abstract}

Copyright (C) EPLA, 2007

Introduction. - The transport process of aggregates generated in fluids has been the subject of numerous studies ranging from basic to applied sciences [1], including biophysics [2]. The scaling relation between settling velocities $u_{a}$ and sizes $a$ of aggregates such as $u_{a} \sim a^{\beta}$ has been confirmed by settling experiments under the gravitational force [3-8]. In some cases, fractal dimensions $D_{f}$ obtained by using Stokes' law $u_{a} \sim \Delta \rho(a) a^{2}$ are in agreement with those determined by direct observations [9]. We need, however, caution when applying Stokes' law to permeable porous fractal-systems since it is in principle applicable to impermeable systems. Many theoretical attempts have been made to elucidate the interior flow of fractal aggregates [10-16]. Most of works have treated the hydraulic permeability as a function of the porosity $P$, ignoring the characteristics of fractal structures.

In this letter, Brinkman equations are employed to obtain the analytic expression for the settling process of fractal aggregates in water. According to the close analogy between the electrical conductance and the fluid flow in porous fractal-media, we consider the effect of permeant flow through connected (backbone) channels in aggregates on the settling velocity. Our analysis gives theoretical foundation that the scaling relation between the settling

\footnotetext{
(a) E-mail: tnaka@eng.hokudai.ac.jp

(b) E-mail: jphuang@fudan.edu.cn
}

velocities and the sizes of aggregates holds. In addition, we demonstrate that Stokes' law is applicable under certain circumstances to derive fractal dimensions of aggregates generated in water.

Theoretical analysis. - A feature of settling of aggregates is that streamlines traverse porous aggregates. The Brinkman equations are established to describe the permeant flow [17], where the porosity-dependent permeability $k$ is introduced for describing the permeant flow in a spherical aggregate of radius $a$. The Brinkman equations are given by the following set of equations:

$$
\begin{aligned}
& \nabla p_{i}=\mu \nabla^{2} \mathbf{u}_{i}-\frac{\mu}{k}\left(\mathbf{u}_{i}-\mathbf{u}_{a}\right) ; \quad r \leqslant a, \\
& \nabla \cdot \mathbf{u}_{i}=0
\end{aligned}
$$

where $p_{i}$ denotes the pressure, $\mathbf{u}_{i}$ the velocity of fluid inside an aggregate, $\mathbf{u}_{a}$ the velocity of the aggregate, $\mu$ the kinetic viscosity of a fluid, respectively. The governing equation for the flow outside of an aggregate is given by Stokes equations, $\nabla p_{o}=\mu \nabla^{2} \mathbf{u}_{o} ; r \geqslant a$, and $\nabla \cdot \mathbf{u}_{o}=0$, where the subscripts $o$ defines outside an aggregate. The vorticity is defined as $\mathbf{w}_{i}=\nabla \times \mathbf{u}_{i}, \mathbf{w}_{o}=\nabla \times \mathbf{u}_{o}$ inside and outside of an aggregate, respectively. For convenience, we formulate the problem in spherical coordinates $[r, \theta, \varphi]$. Since the flow is axial-symmetric, the fluid velocity in the $\varphi$ direction is zero. Furthermore, we introduce the 
conventional stream functions [18] defined by

$$
\begin{aligned}
& u_{r}=\frac{1}{r^{2} \sin \theta} \frac{\partial \psi}{\partial \theta} \\
& u_{\theta}=-\frac{1}{r \sin \theta} \frac{\partial \psi}{\partial r} .
\end{aligned}
$$

These expressions describe the behaviour of the flow of water inside and outside the aggregate.

The boundary conditions imposed are: i) the fluid velocity is zero far from the aggregate $(r \rightarrow \infty)$, ii) the normal and tangential components of the velocity vector and stress tensor are continuous at the outer boundary of the porous aggregate $(r \rightarrow a)$, and iii) the normal and tangential components of the velocity vector on the surface of the primary particle at the center $\left(r \rightarrow a_{0}\right)$ are equal to that of the aggregate $\mathbf{u}_{a}$ (no slip condition). These can be expressed such as $\left.\mathbf{u}_{o}\right|_{r \rightarrow \infty}=0,\left.\mathbf{u}_{o}\right|_{r \rightarrow a}=$ $\left.\mathbf{u}_{i}\right|_{r \rightarrow a},\left.\partial\left(\mathbf{u}_{o}\right)\right|_{r \rightarrow a}=\left.\partial\left(\mathbf{u}_{i}\right)\right|_{r \rightarrow a}$, and $\left.\mathbf{u}_{i}\right|_{r \rightarrow a_{0}}=\mathbf{u}_{a}$, where $\partial$ means the space partial of the velocity. Assuming that the permeability $k$ varies slowly and that the velocity of the aggregate has only the vertical component $u_{a} \hat{\mathbf{z}}$, the solutions to stream functions under the above boundary conditions are obtained as

$$
\begin{aligned}
& \psi_{i}=\frac{k u_{a} \sin ^{2} \theta}{2} \frac{1}{\xi}\left[3(1+\xi) \frac{-\cosh \xi+\sinh \xi}{\operatorname{coth} \xi_{1}+\xi_{2} \sinh \xi_{1}}+\xi^{3}\right], \\
& \psi_{o}=\frac{k u_{a} \sin ^{2} \theta}{2} \frac{1}{\xi} \\
& \times\left[3\left(1-\frac{\xi^{2}}{2}+\frac{\xi_{1}^{2}}{2}\right)\left(\frac{\xi_{2}+\tanh \xi_{1}}{1+\xi_{2} \tanh \xi_{1}}+\xi_{1}\right)-\xi_{1}^{3}\right],
\end{aligned}
$$

where $\xi=r / \sqrt{k}, \xi_{1}=a / \sqrt{k}$, and $\xi_{2}=a_{0} / \sqrt{k}$, respectively.

With the use of eq. (3), the drag force, $F_{d}$, exerted by the fluid on the aggregate can be calculated by integrating the stress over the surface of the aggregate,

$$
F_{d}=-6 \pi \mu u_{a} a+6 \pi \mu u_{a} \frac{\sqrt{k}\left(a_{0}+\sqrt{k} \tanh (a / \sqrt{k})\right)}{a_{0} \tanh (a / \sqrt{k})+\sqrt{k}} .
$$

Equation (4) is a modification of Stokes' law for a porous aggregate. In it, for an isolated particle $(k \rightarrow \infty)$, the correction factor approaches the value $a_{0}$, indicating that the drag force $F_{d}$ can be negligible for such a small particle.

The settling velocity is calculated from a force balance. There are three forces, gravity $\left(F_{g}\right)$, buoyant $\left(F_{b}\right)$, and drag $\left(F_{d}\right)$, acting upon an aggregate. The sum of the gravity and buoyant forces equals the drag force $F_{d}$ such as $F_{g}-F_{b}=F_{d}$. If all particles constituting an aggregate have the same density $\rho_{a}$, and the suspending water density is $\rho_{l}$, the drag force can be expressed by

$$
F_{d}=\frac{4 \pi}{3} a^{3}\left(\rho_{a}-\rho_{l}\right) g=\frac{4 \pi}{3} a^{3}(1-P) \Delta \rho g,
$$

where $P$ is the aggregate porosity and $\Delta \rho$ is the difference of mass densities between a primary particle and fluid. Using eqs. (4) and (5), we obtain the modified Stokes law given by

$$
\begin{aligned}
u_{a}= & -\frac{2 a^{2} g(1-P) \Delta \rho}{9 \mu} \\
& \times \frac{a a_{0} \tanh (a / \sqrt{k})+\sqrt{k} a}{\sqrt{k}\left(a_{0}-a\right)+\left(k-a a_{0}\right) \tanh (a / \sqrt{k})} .
\end{aligned}
$$

Here the hydraulic permeability $k$ is a key parameter to describe the permeant flow in porous fractal-aggregates. For impermeable spherical aggregates, the settling velocity is proportional to the aggregate cross-section $a^{2}$ according to Stokes' law, but permeable aggregates show different behaviours as demonstrated experimentally [3-8].

Fractality of aggregates. - For a fractal aggregate with the fractal dimension $D_{f}$, the scaling law between the aggregate size $a$ and the total mass $M$ holds for [19]

$$
M \sim N \sim a^{D_{f}}
$$

where $N$ is the number of primary particles forming the aggregate. As a consequence of the fractal geometry of aggregates, we have from eq. (7) the following relation for the porosity:

$$
1-P=\frac{N v_{0}}{V_{a}}=\left(1-P_{0}\right)\left(\frac{a}{a_{0}}\right)^{D_{f}-3}
$$

where $v_{o}$ and $V_{a}$ are the volumes of a single particle and an aggregate, respectively, and $P_{0}$ is defined as a porosity at $a=a_{0}$, namely, $P_{0}=0$.

Numerous theoretical works have been made by supposing constant permeabilities. However, this assumption is obviously unreasonable for fractal aggregates, because the permeability $k$ should reflect the fractality of aggregates. Some considerations can be made according to the dimension analysis. The permeability of aggregates has to satisfy the following extreme conditions: i) a completely porous aggregate $(P=1)$ indicates full-of-fluid flow in the aggregate, and the permeability will tend to infinity, ii) the permeability should have a critical value $k_{c}\left(P_{c}\right)$, below which the permeability should equal to zero reflecting non-transparent situations, and iii) the permeability critical exponent $t$ should be defined through the relation $k \sim\left(P-P_{c}\right)^{t}$ since we can map the problem exactly onto that of the electrical conduction problem, with the electrical current density replacing the fluid current density, and electrical field replacing the fluid pressure gradient [20]. Thus, we can define the permeability exponent $t$ as the conductivity exponent.

We should note that the flow through aggregates occurs via the connected (backbone) channels of the fractal aggregates, and dead ends attached to the channels carry no fluid flow [20]. The situation is identical to the problem 
of electrical conductance through fractal networks, where only connected (backbone) channels contribute to the electrical conductance. Thus, the fluid flow traverses only along connected backbone-channels in fractal aggregates. Actually, we can view our fractal aggregates as composites consisting of solid and fluid components, which are identical to the case of percolative metal/insulator composites; namely, we can relate to the conductivity problem for such systems. From these considerations i)-iii), we can express the criticality of the permeability as

$$
k=d^{2} \frac{\left(P-P_{c}\right)^{t}}{1-P}, \quad P \geqslant P_{c},
$$

where $d$ is a characteristic length scale and $t$ is the conductivity exponent, respectively.

Attempts have been made to obtain approximate expressions for the permeability $k$ [10-17,21]. Among these, the expression obtained by Brinkman [17] for swarms of particles only satisfies three conditions i)-iii) for the permeability $k$ mentioned below eq. (8). This is given by

$$
k=\frac{1}{18} a_{0}^{2}\left(-3 \sqrt{\frac{8}{1-P}-3}+\frac{4}{1-P}+3\right) .
$$

At first glance, this equation seems not to satisfy the conditions i)-iii) for $k$. In addition, eq. (6) combined with eq. (10) seems not to recover the scaling relation observed experimentally. We have found, however, that eq. (10) can be expanded in a power series at $P_{c}=1 / 3$ such that

$$
\begin{aligned}
k= & \frac{a_{0}^{2}}{1-P}\left[\frac{1}{6}\left(P-P_{c}\right)^{2}+\frac{1}{12}\left(P-P_{c}\right)^{3}\right. \\
& \left.+\frac{1}{12}\left(P-P_{c}\right)^{4}+\ldots\right] .
\end{aligned}
$$

We should note that the leading term in eq. (11) behaves at around $P_{c}=1 / 3$ such as

$$
k=\frac{a_{0}^{2}\left(P-P_{c}\right)^{2}}{6(1-P)}
$$

Comparing eqs. (9) and (12), we realize that our hypothesis eq. (9) is quite reasonable by putting $d^{2} \approx a_{0}^{2} / 6$. Equation (12) indicates the conductivity exponent to be $t=2$. In addition, we should note that the effective medium approximation (EMA) by Bruggeman [22,23] predicts $P_{c}=1 / D$, where $D$ is the Euclidean dimensionality and $t=1$ for percolative metal-insulator composites. It is remarkable that eq. (11) is expanded around the value $P_{c}=1 / 3$, the same as Bruggeman's $P_{c}=1 / D$.

Comparisons with experiments. - Many experiments have been performed on the sedimentation of fractal aggregates [3-8]. For instance, the experiments made by Johnson et al. [5] have used aggregates generated from latex microspheres about several hundreds $\mu \mathrm{m}$ in diameter with a density of $1.05 \mathrm{~cm}^{-3}$. Microspheres (2.5\% suspension in weight) were coagulated in $\mathrm{NaCl}$ solutions. They employed three kinds of aggregates with fractal dimensions $D_{f}=1.79,2.19$, and 2.25 , respectively, in which the fractal dimensions were derived from the direct observation of size-weight relationship [5]. We use these parameters to compare our numerical results with experimental data on settling velocities of fractal aggregates. It should be noted that these values of the fractal dimension $D_{f}$ are close to those of fractal aggregates generated by diffusionlimited cluster-cluster process $(\mathrm{DLCA})\left(D_{f} \approx 1.78\right)$ and the value of the reaction-limited cluster-cluster process $(\mathrm{RLCA})\left(D_{f} \approx 2.1\right)[24]$.

We should consider the effect of pressure gradient for the formation of aggregates in water due to the gravitational force. There is a possibility to form vertically directed (anisotropic) aggregates under gravitational force. If this is the case, a different value of the conductivity exponent $t$ from that of equilibrium isotropic aggregates is required [25]. About this point, the settling velocities of fractal aggregates in water are proportional to the difference of mass densities between aggregates and water $\Delta \rho(a)=\rho_{a}-\rho_{l}$. The aggregates we are interested in have the mass densities close to that of water, so $\Delta \rho$ takes very small values between 0.1 and $0.01 \mathrm{~g} / \mathrm{cm}^{3}$. This indicates that settling velocities of aggregates are quite small. In fact, the observed settling velocities range from 0.5 to $0.01 \mathrm{~cm} / \mathrm{s}$. This indicates that we can neglect the gravitation effect to the formation of aggregates in water, implying that it is sufficient to take into account the exponent $t$ for isotropic and equilibrium aggregates. However, within our knowledge, there is no theoretical prediction on the value of the conductivity exponent $t$ for fractal aggregates though the spectral dimensions $d_{s}$ are obtained for DLCA and RLCA [26]. So, we employ the known values of the conductivity exponents of $t$ for our aggregates from $D=2$ percolation network of $D_{f}=1.89 \ldots$ and $D=3$ percolation network of $D_{f}=2.48 \ldots$, since these values of $D_{f}$ are close each other. Namely, we compare numerical results with experimental data on settling velocities of fractal aggregates by taking $t=1.264$ for the aggregate of $D_{f}=1.79$ and $t=1.867$ for the aggregate of $D_{f}=2.19$ and 2.25, respectively [27].

Numerical and experimental results are given in fig. 1 in terms of a log-log scale because we are only interested in the power law behaviour of the settling processes. Actually, the absolute value of the calculated settling velocity is still slower than the experiment data. It will be seen from fig. 1 that a good agreement is obtained between our calculations and experiments, in particular, for dense systems such as $D_{f}>2$. Further details are displayed in table 1 in which numerical results based on eqs. (6) and (9) are listed.

We should emphasize that dense aggregates with $D_{f}>2$ correspond to low permeable cases of $k \ll a^{2}$, where the fluid mainly flows via the outer region of aggregates. Using the approximation of $\tanh (a / \sqrt{k}) \approx 1$ for $k \ll a^{2}$ in eq. (6), 

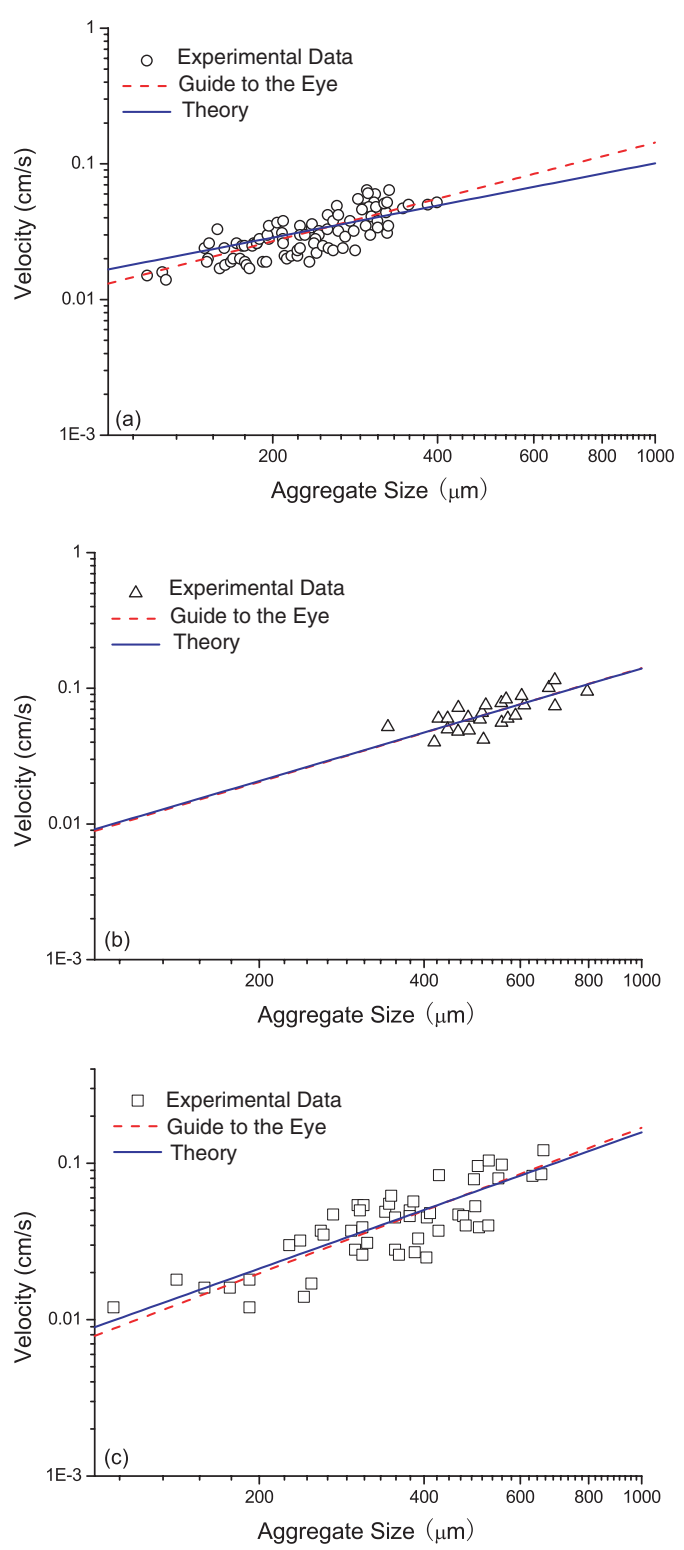

Fig. 1: Settling velocities of aggregates vs. aggregate sizes. The scatters are experimental data taken from ref. [5]. The dashed lines are fitted to experimental data for a guide to the eye. The solid lines are the calculated results according to our theory (eq. (9)) for the cases of (a) $D_{f}=1.79$, (b) $D_{f}=2.19$, (c) $D_{f}=2.25$, respectively. The absolute values of velocities are omitted to give prominence to the power law.

Table 1: The scaling relation $u_{a} \sim a^{\beta}$ between settling velocities and sizes of aggregates. Comparisons are made between experiments [5] and our theory taking fractal dimensions $D_{f}$ as a parameter.

\begin{tabular}{lll}
\hline$D_{f}$ (Exper) & $\beta$ (Exper) & $\beta$ (Theory) \\
\hline 1.79 & $1.04 \pm 0.10$ & 0.78 \\
2.19 & $1.20 \pm 0.11$ & 1.19 \\
2.25 & $1.33 \pm 0.10$ & 1.25 \\
\hline
\end{tabular}

we have the expression for the scaling relation of the form

$$
u_{a} \sim a^{D_{f}-1}, \quad \text { for } k \ll a^{2} .
$$

Thus, we can derive fractal dimensions $D_{f}$ of aggregates using eq. (13) for low permeable cases, from which the fractal dimensions are obtained as $D_{f}=2.04,2.20$, and 2.33 for three kinds of aggregates used in experiments. These fractal dimensions $D_{f}$ are in agreement with $D_{f}$ 's obtained from direct observations, especially for desnse systems $D_{f}>2$.

Conclusions. - We have made a theoretical study about the settling process of aggregates generated in water. Our calculated results on settling velocities clearly show the scaling relation $u_{a} \sim a^{\beta}$ between settling velocities and sizes of aggregates and fit remarkably well with experimental data. The analytic expression for the settling velocity provides a criterion for determining fractal dimensions $D_{f}$ of aggregates from sedimentation experiments. The procedure proposed in this letter can be used to characterize the interior fluid flow even in other types of porous media.

This work was supported by the Grant-in-Aid for Scientific Research by the Japan Ministry of Education, Science, and Culture. JPH and WJT acknowledge the financial support from the National Natural Science Foundation of China under Grant No. 10604014, the Shanghai Education Committee and the Shanghai Education Development Foundation ("Shu Guang" project), and the Pujiang Talent Project (No. 06PJ14006) of the Shanghai Science and Technology Committee. KWY acknowledges financial support from the RGC Earmarked Grant of the Hong Kong SAR Government.

\section{REFERENCES}

[1] For a review see, for example, Bushell G. C., YAN Y. D., Woodfield D., Raper J. and Amal R., Adv. Colloid Interface Sci., 95 (2002) 1 and references therein.

[2] Tuinier R., Dhont J. K. G. and FAn T. H., Europhys. Lett., 75 (2006) 929.

[3] Tambo N. and Watanabe Y., Water Res., 13 (1979) 409. The original versions in Japanese were published in Suido Kyokai Zasshi, 397 (1967) 2; 410 (1968) 14; 445 (1971) 2.

[4] Li D. H. and Ganczarczyk J., Environ. Sci. Technol., 23 (1989) 1385.

[5] Johnson C. P., Li X. Y. and Logan B. E., Environ. Sci. Technol., 30 (1996) 1911.

[6] Lee D. J., Chen G. W., Liao Y. C. and Hsieh C. C., Water Res., 30 (1996) 541.

[7] Gregory J., Water Sci. Technol., 36 (1997) 1.

[8] Li X. Y., YuAN Y. and Wang H. W., Environ. Sci. Technol., 37 (2003) 292.

[9] Meakin P., Adv. Colloid Interface Sci., 28 (1988) 249.

[10] Neale G., Epstein N. and Nader W., Chem. Eng. Sci., 28 (1973) 1865. 
[11] Howells I. D., J. Fluid Mech., 64 (1974) 449.

[12] Neale G. and Nader W. K., AIChE J., 20 (1974) 530.

[13] Adler P. M., J. Colloid Interface Sci., 81 (1981) 531.

[14] Rogak S. N. and Flagan R. C., J. Colloid Interface Sci., 134 (1990) 20.

[15] Veerapaneni S. and Wiesner M. R., J. Colloid Interface Sci., 177 (1996) 45.

[16] Wu. R. M. and LeE D. J., Water Res., 32 (1998) 760.

[17] Brinkman H. C., Appl. Sci. Res. A, 1 (1947) 27; (1947) 81; 2 (1948) 190; Research (London), 2 (1949) 190.

[18] Happel J. and Brenner H., Low Reynolds Number Hydrodynamics (Kluwer, Dordrecht) 1991.

[19] See, for example, Nakayama T. and Yakubo K., Fractal Concepts in Condensed Matter Physics (Springer-Verlag, Berlin) 2003.
[20] De Gennes P. G., J. Phys. (Paris) Lett., 37 (1976) L1; J. Fluid Mech., 136 (1983) 189.

[21] Matsumoto K. and Suganuma A., Chem. Eng. Sci., 32 (1977) 445; Matsumoto K. et al., Chem. Eng. Sci., 33 (1978) 1554.

[22] Bruggeman D. A. G., Ann. Phys. (Leipzig), 24 (1935) 636.

[23] Huang J. P. and Yu K. W., Phys. Rep., 431 (2006) 87.

[24] See, for example, VicseK T., Fractal Growth Phenomena (World Scientific, Singapore) 1992.

[25] For a review see, for example, Loscar E. and Albano E. V., Rep. Prog. Phys., 66 (2003) 1343.

[26] Terao T. and Nakayama T., Physica B, 263-264 (1999) 317.

[27] Nakayama T., Yakubo K. and Orbach R., Rev. Mod. Phys., 66 (1994) 381 and references therein. 